\title{
Nonlinear orientation effect in liquid crystals to create a linear displacement sensor
}

\author{
Olga Denisova ${ }^{1, *}$, Roman Abramishvili ${ }^{2}$ \\ ${ }^{1}$ Department of Physics, Ufa State Petroleum Technological University, Kosmonavtov Str., 1, Ufa, 450062, Republic of Bashkortostan, \\ Russia \\ ${ }^{2}$ Department of Management and Service in Technical Systems, Ufa State Petroleum Technological University, Kosmonavtov Str.,1, \\ Ufa, 450062, Republic of Bashkortostan, Russia
}

\begin{abstract}
The results of experimental studies of thin layers of nematic liquid crystal under the influence of low frequency periodic oscillation (frequency about $100 \mathrm{~Hz}$ ) are presented in this work. Before reaching the critical impact of the liquid crystal director varies symmetrically with respect to the normal to the cell. Further, when the oscillation amplitude is 0,8 microns, the liquid crystal molecules tilt relative to the normal to the cell while continuing to oscillate. The results of this research can be used in the development of liquid crystal sensors for diagnostics of mechanical displacements.
\end{abstract}

\section{Introduction}

Despite the fact that liquid crystals were discovered more than 100 years ago, they still cause great interest in the scientific community. Liquid crystals (LC) are a state of matter intermediate between a liquid and a solid. This state has a many ranges of practical applications. In the words of P. de Gennes, "Liquid crystals are beautiful and mysterious. I admire both". First of all, the liquid crystals have accelerated the revolution in the art of visual presentation devices (displays), and it is of interest to those involved in basic research. The second, the liquid - crystalline state inherent to many biologically active systems, including the human body, and therefore is one of the most important areas of biological research. But, unfortunately, until now, many of the fundamental problems remain unresolved.

Nematics and cholesterics are very sensitive to external fields. The first effects (due to the influence of the magnetic field) were found in the 30's and 40's of last century, by a group of Soviet scientists headed by V.K. Frediriks and V.N. Tsvetkov. Recently, interest increased to liquid crystals as nanomaterials. The size of the liquid crystal molecules is from a thickness of $5 \AA$ and $30 \AA$ in length to $200 \AA$ in thickness and a length of $3000 \AA$, for example, tobacco mosaic virus. Biological membranes - a thin $(\sim 80 \AA)$ from the leaves of lipids and proteins. They play a key role in many life processes, but their structure is little known. Most physical experimentation (for example, NMR) may not be carried on a separate membrane because it contains too little substance. However, one can create a model system of water and lipids or even of lipid, protein and water, having a lamellar (layered) structure. It is assumed that each individual layer is the analogue membrane.

The aim of the present scientific work was to the discover and investigate the stability of orientation, which is observed in the mechanical vibrations. The effect has a threshold of occurrence. It occurs due to violation of the spatial symmetry of the LC - system. LC procedure affects many of the physical properties of matter, and therefore the liquid crystals can be investigated by various experimental methods: ultrasonic, nuclear magnetic resonance, electron paramagnetic resonance, methods of linear and nonlinear optics. A new method has been proposed and developed for the detection and experimental study of the effects mentioned above.

The influences of external factors (acoustic, electric, magnetic, temperature fields) to the liquid crystals accompany by a variety of effects and structural changes in a wide spectral range $10^{-1} \leq \omega \leq 10^{8} \mathrm{~Hz}$. According to numerous visual observations, there is three stages of changing the structure of oriented sample: homogeneous, spatially periodic and non-uniform deformation.

A comparison of the characteristics of the different acousto-optic effects in liquid crystals in the presence of a temperature gradient, electric or magnetic fields, and without them, gives reason to conclude that the basis of the effects caused by the periodic shear deformation is the same physical mechanism.

Obviously, the presence of vibrations is characteristic of mechanisms containing moving elements. If the permissible readings are exceeded, an accident or equipment failure can occur [1-3]. Recently, nondestructive testing methods have been actively introduced, which provide timely detection of problems in equipment. Vibration is one of the most accessible and giving the necessary information parameters, both for a

\footnotetext{
* Corresponding author: denisovaolga $@$ bk.ru
} 
comprehensive assessment of the technical condition of equipment, and for individual units and aggregates [4 7]. Timely detection of technical problems, including vibration parameters, reduces the costs of repair and maintenance of equipment. They make it possible to solve such technical and economic problems as monitoring the current state of units and assemblies, preventing accidents and failures, as a consequence of preventive repairs while reducing indicators, bringing equipment to repair in accordance with its technical condition, which will increase the inter-repair interval.

To solve similar problems in some branches of science and technology, increased sensitivity and speed are required, and this requires new scientific and technical solutions.

Now there is a large number of instruments and systems for determining vibration parameters, which are based on various physical effects and phenomena [8-20].

The authors propose to create a highly effective system for determining the vibration parameters in nodes and mechanisms based on the acousto-optic effect in liquid crystals of the nematic type described in the article.

The development of this system would give an increase in sensitivity (up to $0,1 \mu \mathrm{m}$ ) and an increase in speed due to a pronounced orientation effect in liquid crystals (the relaxation time is $10^{-6} \mathrm{~s}$ ).

\section{Method}

The experimental data and theoretical development for the high frequency range $(1 \mathrm{MHz}<\omega<100 \mathrm{MHz})$ are mainly reflected in the available scientific papers. The low frequency range $(20 \mathrm{~Hz}<\omega<20 \mathrm{kHz})$ external influences are rather poorly investigated. Thus, the current study is the instabilities that occur when exposed to the liquid crystal of low-frequency periodic shift and their practical use.

Rayleigh (Strutt) John William, L.D. Landau and E.M. Lifschitz (the course of theoretical Physics [6]) considered the motion, which occurs in a viscous fluid with fluctuations immersed in it of solids, which has a number of unique features. The quickly damped viscous shear wave occurs in an isotropic liquid under oscillatory motion with frequency $\omega$ and amplitude $a$, which penetrates to a depth $\delta$.

To a nematic liquid crystal (NLC) with an initial homeotropic orientation of the director, the oscillating body performs periodic oscillations with a frequency $\omega$ and a maximum amplitude $a$. In the linear approximation of the equations of motion of the director $n$ and the molecular centers of inertia can be written as [7]:

$$
\begin{gathered}
K_{33} \frac{\partial^{2} \theta}{\partial z^{2}}-\eta_{4} \frac{\partial \theta}{\partial t}+\eta_{1} \frac{\partial v_{x}}{\partial z}=0 \\
\rho \frac{\partial v_{x}}{\partial t}+\eta_{1} \frac{\partial^{2} \theta}{\partial z \partial t}-\eta_{3} \frac{\partial^{2} v_{x}}{\partial z^{2}}=0
\end{gathered}
$$

The solution can be written in a general form:

$$
\begin{gathered}
v \approx v_{o} \exp \left[-\frac{\sqrt{2}}{2} q(z-\delta)\right] \cos \left[\frac{\sqrt{2}}{2} q(z-\delta)\right] \cos \omega t, \\
\theta \approx \frac{\rho v_{o}}{\eta_{1} q} \exp \left[-\frac{\sqrt{2}}{2} q(z-\delta)\right] \sin \left[\frac{\sqrt{2}}{2} q(z-\delta)\right] \cos \omega t, \\
\sigma_{z y} \approx\left(\alpha_{4}+\alpha_{6}\right) \frac{\partial v_{y}}{\partial z}+\alpha_{3} \frac{\partial \theta}{\partial t}
\end{gathered}
$$

at $\left.\theta\right|_{z=0}=0-<\sigma_{z y} u>\approx \frac{u_{o}^{2}}{2} \sqrt{\frac{\omega\left(\alpha_{4}+\alpha_{6}\right) \rho}{2}}$.

If the boundary conditions for $\theta$ weak, everything is determined by the condition of rapid energy dissipation. It is determined by the thermal conductivity $\chi_{\perp}<<\chi \|$, and that is why in the bed set the transition from $n_{z}$ to $n_{x}$, and whose laws will be examined below.

The movement of the oscillating body (movable plate) in the NLC, leading to heating, will change the order parameter to the threshold of thermal convection. It will lead to instabilities of the liquid crystal orientation.

The object of study of this scientific work was a nematic liquid crystal $n$ - type - methoxybenzylidene - $n$ - butylaniline with a temperature range of mesophase existence of $18-42{ }^{\circ} \mathrm{C}$. The main method of study was the method of interferometry birefringence based on a polarization microscope $[8,9]$. The cell consists of a double sandwich (a massive two glass plates, where between was placed a third glass plate thickness of the cover glass, less than the distance between the massive plates). The record of the cover glass was free to oscillate in its (horizontal) plane. To create a homeotropic orientation of the LC molecules, chromium metal was sputtered on the substrate.

For registration and initial processing of signals studied experimental setup was assembled, where the basic elements of the recording were a polarization optical microscope Amplival Pol $U$ console and spectrophotometric. To create an acoustic contact, membrane of the vibrator was connected by a waveguide. Measuring of the amplitude of the vibrations of thin cell substrate is held by inductive method [9].

According to the experimental procedure of sample preparation and introduction of the acoustic wave in the sample, we have a two layer system on the LC - film thickness $h_{c}$. Effect of acoustic wave on such a system gives rise to birefringence. Moreover, the polarizer and analyzer are crossed and form $45^{\circ}$ with the direction of the shift. Constant intensity $I_{c}$ as well as alternating signal components $I(t)$ in time, having a frequency range of harmonic excitation $\omega$ are recorded.

For the intensity of the optical signal, according to the Malus law:

where $\eta_{1}, \eta_{3}, \eta_{4}$ - coefficient of viscosity. 


$$
\begin{aligned}
& I=I_{o}\left\{\left[\sin ^{2} \frac{\pi h b}{\lambda}<\sin ^{2} \theta_{c}>+\frac{\pi h b}{\lambda}\left[2<\sin \theta_{c} \theta_{t}>+\right.\right.\right. \\
& \left.+<\theta_{t}^{2}>\right] \sin 2 \frac{\pi h b}{\lambda}<\sin ^{2} \theta_{c}>+\left[\frac{\pi h b}{\lambda}\right]^{2}\left[2<\sin \theta_{c} \theta_{t}>+\right. \\
& \left.\left.+<\theta_{t}^{2}>\right]^{2} \cdot \cos ^{2} \frac{\pi h b}{\lambda}<\sin ^{2} \theta_{c}>\right\},
\end{aligned}
$$

where $\lambda$ is the length of the incident light wave, the constant $b=0.23, \theta_{\mathrm{c}}$ is the constant of the component of the angle of inclination of the molecules of the liquid crystal (director), $\theta_{\mathrm{t}}$ is the variable component of the slope angle of the LC molecules.

Components of the optical signal:

$$
\begin{aligned}
& I_{c}=I_{o}\left\{\sin ^{2} \frac{\pi h b}{\lambda}<\sin ^{2} \theta_{c}>+\frac{\pi h b}{\lambda} \frac{\theta_{o}^{2}}{2} \sin 2 \frac{\pi h b}{\lambda} .\right. \\
& \cdot<\sin ^{2} \theta_{c}>+2\left[\frac{\pi h b}{\lambda}\right]^{2}<\sin \theta_{c} \theta_{o}>^{2} \cos ^{2} \frac{\pi h b}{\lambda} . \\
& \left.\cdot<\sin ^{2} \theta_{c}>+\frac{3}{8}\left[\frac{\pi h b}{\lambda}\right]^{2}\left(\theta_{o}^{2}\right)^{2} \cos ^{2} \frac{\pi h b}{\lambda}<\sin ^{2} \theta_{c}>\right\}, \\
& I_{1 \omega}=I_{o}\left\{2 \frac{\pi h b}{\lambda}<\sin \theta_{c} \theta_{o}>\sin 2 \frac{\pi h b}{\lambda}<\sin ^{2} \theta_{c}>+3\left[\frac{\pi h b}{\lambda}\right]^{2} .\right. \\
& \left.\cdot<\sin \theta_{c} \theta_{o}>\theta_{o}^{2} \cos ^{2} \frac{\pi h b}{\lambda}<\sin ^{2} \theta_{c}>\right\} \sin ^{2} \omega t, \\
& I_{2 \omega}=I_{o}\left\{-\frac{\pi h b}{\lambda} \frac{\theta_{o}^{2}}{2} \sin 2 \frac{\pi h b}{\lambda}<\sin ^{2} \theta_{c}>-2\left[\frac{\pi h b}{\lambda}\right]^{2} .\right. \\
& \cdot<\sin \theta_{c} \theta_{o}>^{2} \cos ^{2} \frac{\pi h b}{\lambda}<\sin ^{2} \theta_{c}>-\left[\frac{\pi h b}{\lambda} \frac{\theta_{o}^{2}}{2}\right]^{2} . \\
& \left.\cdot \cos ^{2} \frac{\pi h b}{\lambda}<\sin ^{2} \theta_{c}>\right\} \cos 2 \omega t .
\end{aligned}
$$

Indignation weakly damps on the thickness of the cell, and captures all of the liquid crystal layers in this frequency range.

According to formula (5), the signal recorded at the excitation frequency $\omega$, occurs only when there is a stationary tilt $\theta_{s}$ director and the normal to the surface, i.e. in violation of symmetry LC - the system relative to the normal to the layer. The appearance of the AC signal on the disturbance frequency determines a value of the threshold of an acoustic wave amplitude in the NLC, leading to the formation of a stationary director tilt in one direction or another deflection of the vibrating plate.

\section{Result and discussion}

According to the experimental data, the behaviors of the variable optical component signal $I_{c}$ (a) and the second harmonic $I_{2 \omega}(a)$ (Figure 1), the mean square amplitude of the sine components constant director tilt $<\sin ^{2} \theta_{c}>(a)$ and the variable part $\left\langle\theta_{0}^{2}\right\rangle$ (a) are calculated (Figure 2).

a)

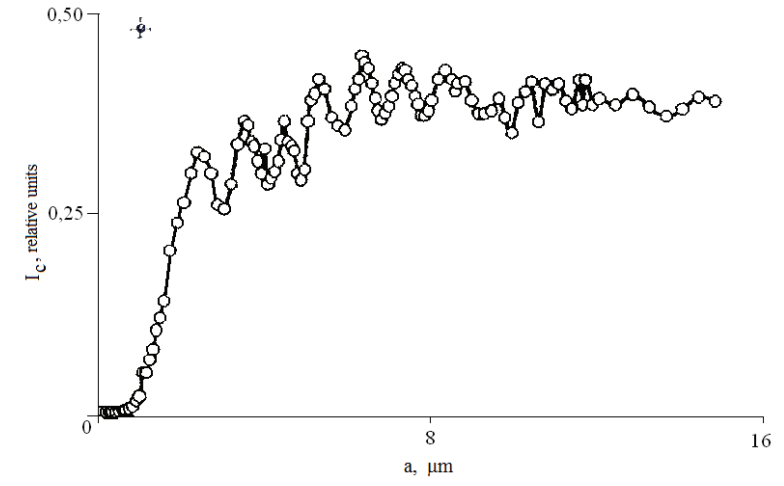

b)

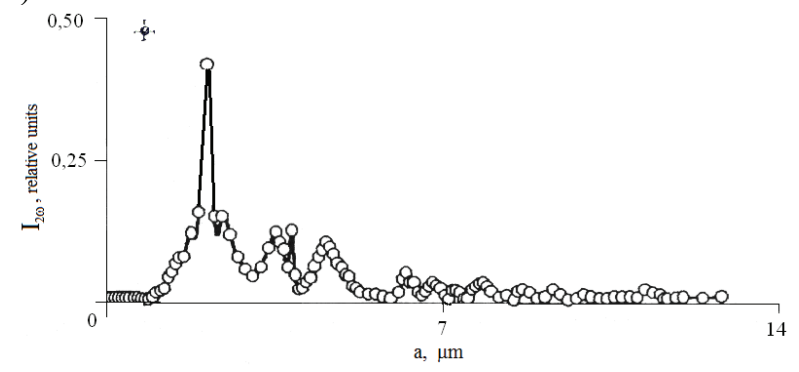

Fig. 1. Amplitude dependences of a) constant component of the optical signal $I_{c}, b$ ) second harmonic optical signal $I_{2 \omega}$.

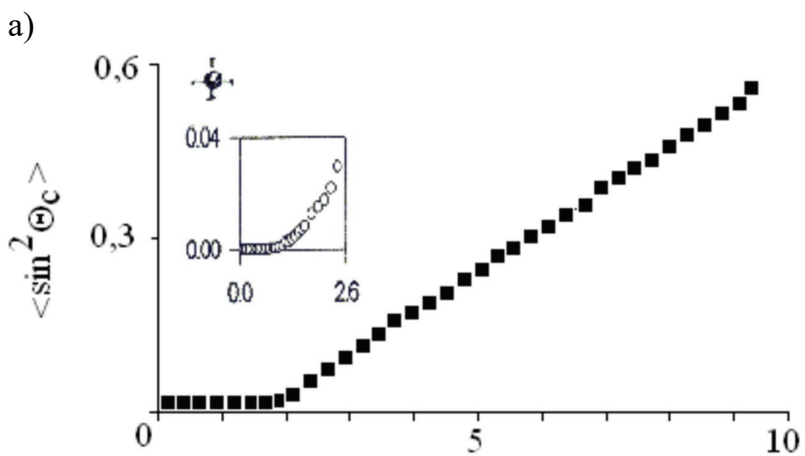

a, $\mu \mathrm{m}$

b)

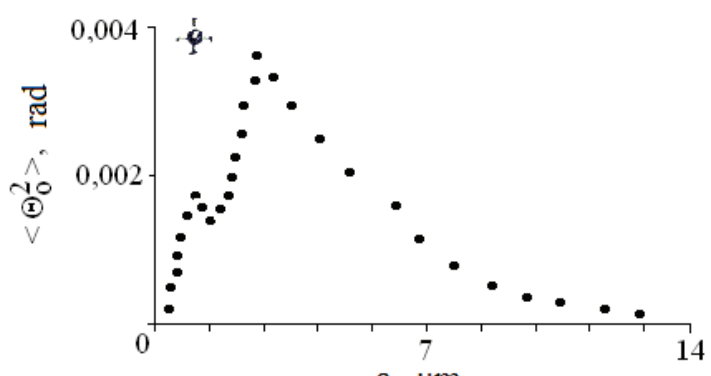

$\mathrm{a}, \mu \mathrm{m}$

Fig. 2. Amplitude dependences of a) mean square of the sine of the angle of inclination of the stationary $<\sin ^{2} \theta_{c}>, b$ ) variable part $<\theta_{0}{ }^{2}>$ of director of the tilt angle.

As we see (Figure 2), the function $\left\langle\sin ^{2} \theta_{c}>(a)\right.$ increases monotonically, tending to the maximum value - to 1 , and $\left.<\theta_{o}^{2}\right\rangle$ (a) reaches a maximum at a certain value of amplitude and, then begins to subside. The increase of the stationary tilt of the director leads to a reduction of the dynamic susceptibility LC - layer. 
The question of the definition of this type of orientation approach in shear wave field is reduced to the study of the frequency dependence of the threshold value an education inpatient director tilt. If $a_{n}(\omega)=$ const, then we can talk about the acoustic analogue of the Fredericks transition [7]. This behavior is confirmed by the data [10]. The observed pattern of behavior homeotropic NLC layer at periodic low-frequency shifts were predicted in theoretical studies $[11,12]$.

Orientation instability of this type leads to permanent changes in time orientation of the molecules. In the case where the wavelength of a viscous layer of considerably greater thickness, it is proportional to the threshold of instability LC thickness layer and does not depend on the strain rate. We have identified a threshold determined to depending delete on the temperature and the thickness of the LC layer. We can say that the threshold forming the stationary director tilt in the temperature range of $18<T<45^{\circ} \mathrm{C}$ and a thickness of $20<h_{c}<125$ microns remains constant ( $a_{n} \approx 0,8$ microns) (Figure 3 ) [13 - 20].

We are invited a new method for controlling the vibrations of the nodes of mechanisms and aggregates. The uniqueness of the method lies in the application of the acousto-optic effect in liquid crystals (LC). The LCD cell serves as an indicator, the light transmission, which depends on the amplitude of the oscillations. Its essence lies in the fact that a monochromatic beam of light, passing through a polarizer, falls on a cell filled with a liquid crystal.

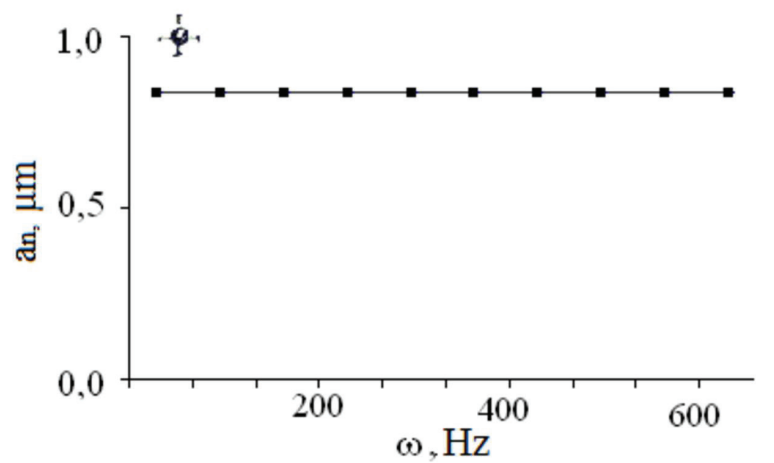

a)

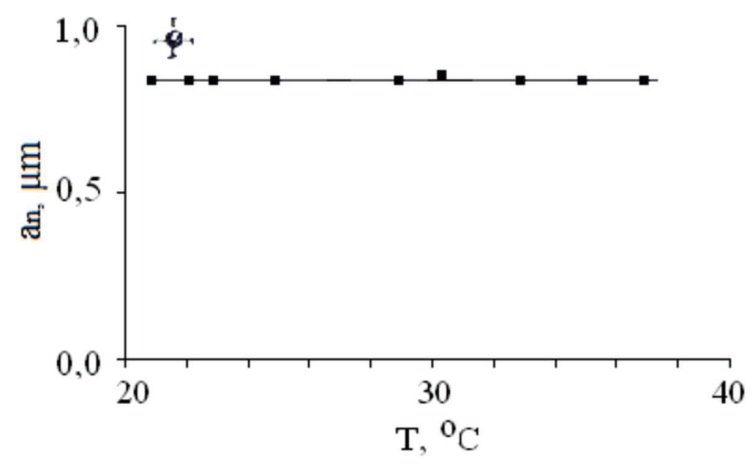

b)

Fig. 3. Dependence of the threshold shift amplitude of an effect the formation of a stationary angle of the director of a) the frequency of exposure, b) temperature.

The cell is designed in such a way that the liquid crystal is between two massive transparent plates separated by spacers. In addition, there is one more thin plate between the plates that can make shear vibrations in the plane of the cell. This plate is connected by a waveguide to a mechanism, the vibration on which it is necessary to control. During its operation, oscillations are transmitted along the waveguide to a moving plate. From it, shear perturbations propagate in a liquid crystal. When the threshold value of the vibration amplitude is reached, initially homeotropically oriented liquid crystal molecules deviate from the normal to the cell. As a result, the light beam incident on the cell passes and is fixed by the photodetector. Further, the processed signal is output to the operator's monitor in a form convenient for analysis.

It should be noted that the occurrence of this acoustooptic effect is of a threshold nature. The threshold value of the amplitude of the action does not depend on the frequency, temperature, thickness of the LC layer. Dependence of the deviation angle of the director on the amplitude of vibration has a linear relationship, which simplifies the calibration of the sensor. The use of a cell on liquid crystals as an indicator will increase the accuracy and sensitivity of the vibration monitoring method. Since liquid crystals are systems more sensitive than solid crystals. The relaxation time of the orientational effects in liquid crystals is $\sim 10^{-6} \mathrm{~s}$.

\section{Conclusion}

In summary, the work carried out showed the spread of transverse vibration of a viscoelastic wave in NLC under the influence of low-frequency periodic shift. It leads to instability of the orientation of nematic liquid crystals. The effect of the formation of the stationary tilt of the director in a homeotropically oriented layers of nematic liquid crystals. It is shown that the amplitude of the critical independence of the orientation of the transition from the frequency shift, the thickness of the crystal temperature. The results presented research can be used in the development of acousto-optic sensors for diagnostics of mechanical vibrations. From the practical point of view, this method is of interest for use in the oil and gas industry and the pulp and aircraft industry.

\section{Reference}

1. V.N. Chelomei (prev.), M.D. Genkina (Ed.) Vibrations in technology: Handbook. In the 6th edition of Ed. Advice, 5 (Mashinostroenie, Moscow, 1981)

2. A.I. Yashura. The system of maintenance and repair of general industrial equipment: a directory (Izd-vo NTs ENAS, Moscow, 2006)

3. F.M. Dimentberg. Flexural vibrations of rotating shafts (Izd. Akad. Nauk SSSR, Moscow, 1959).

4. V.P. Sivakov, V.N. Mikushina, E.N. Stepanova. Modern problems of science and education, 6 (2014) [in Russian]

5. A.I. Morenko, D.V. Tarakanov, Sensors and systems, 3 (2001) 
6. L.D. Landau, E.M. Lifshitz. Theoretical physics, 6 (Gos.isd. fis-mat.lit., Moscow, 1986)

7. De Gennes P., Physics of Liquid Crystals (Mir, Moscow, 1977)

8. O.A. Denisova, Electrical and information complex and systems, 9 (2), 107-113 (2013)

9. O.A. Denisova, The nonequilibrium structural changes of liquid crystals in electric fields and currents acoustic. Scientific publication (UGAES, Ufa, 2012)

10. G.N. Belova, E.I. Remizova, Acoustic magazine, 3, 289-295 (1985)

11. I.A. Chaban, Acoustic magazine, 1, 132-134 (1985)

12. I.A. Chaban, Acoustic magazine, 24, 260-270 (1978)

13. O.A. Denisova. Electronic scientific journal "Oil and gas business", 5 (2011)

14. O.A. Denisova, O.A. Skaldin, Letters on materials, 6 (3), 168-172 (2016)

15. O.A. Baimakova , O.A. Skaldin, A.N. Chuvyrov, Mol. Cryst. Liq. Cryst., 265 (1995)

16. O.A. Denisova, A.N. Chuvyrov, Liq. Cryst. and their Appl., 1 (2012)

17. O.A. Denisova, A.N. Chuvyrov. Liq. Cryst. and their Appl., 2 (2011)

18. O.A. Denisova, A.N. Chuvyrov. Liq. Cryst. and their Appl., 3 (2011)

19. V.V. Leonov, O.A. Denisova, Electrotechnical and Information Systems and Systems, 2 (2015)

20. O.A. Denisova, A.N. Chuvyrov, Liq. Cryst. and their Appl., 2 (44) (2013) 